Supplementary Materials:

\title{
Detection of atmospheric gaseous amines and amides by a high resolution time-of-flight chemical ionization mass spectrometer with protonated ethanol reagent ions
}

Lei Yao ${ }^{1}$, Ming-Yi Wang ${ }^{1}$, Xin-Ke Wang ${ }^{1}$, Yi-Jun Liu ${ }^{1,2}$, Hang-Fei Chen ${ }^{1}$, Jun Zheng ${ }^{3}$, Wei $\mathrm{Nie}^{4,5}$, Ai-Jun Ding ${ }^{4,5}$, Fu-Hai Geng ${ }^{6}$, Dong-Fang Wang ${ }^{7}$, Jian-Min Chen ${ }^{1}$, Douglas R. Worsnop ${ }^{8}$, Lin Wang ${ }^{1,5 *}$

${ }^{1}$ Shanghai Key Laboratory of Atmospheric Particle Pollution and Prevention $\left(L A P^{3}\right)$, Department of Environmental Science \& Engineering, Fudan University, Shanghai 200433, China

${ }^{2}$ now at Pratt school of engineering, Duke University, Durham, NC 27705,USA

${ }^{3}$ Jiangsu Key Laboratory of Atmospheric Environment Monitoring and Pollution Control, Nanjing University of Information Science \& Technology, Nanjing 210044, China

${ }^{4}$ Joint International Research Laboratory of Atmospheric and Earth System Sciences, School of Atmospheric Science, Nanjing University, 210023, Nanjing, China

${ }^{5}$ Collaborative Innovation Center of Climate Change, Nanjing, Jiangsu Province, China

${ }^{6}$ Shanghai Meteorology Bureau, Shanghai 200135, China

${ }^{7}$ Shanghai Environmental Monitoring Center, Shanghai 200030, China

${ }^{8}$ Aerodyne Research, Billerica, MA 01821, USA

*Corresponding Authors: L.W., email, lin_wang@fudan.edu.cn; phone, +86-21-65643568; fax, +86-21-65642080. 
Table S1 Tentative formula assignment of MS peaks with $\mathrm{m} / \mathrm{z}$ values less than $163 \mathrm{Th}$.

\begin{tabular}{|c|c|c|c|}
\hline ID & Ion formula & Molecular weight & Potential identity \\
\hline 1 & $\mathrm{NH}_{3} \cdot \mathrm{H}^{+}$ & 18.0338 & \\
\hline 2 & $\mathrm{H}_{2} \mathrm{O} \cdot \mathrm{H}^{+}$ & 19.0178 & \\
\hline 3 & $\mathrm{HCN} \cdot \mathrm{H}^{+}$ & 28.0182 & Hydrogen cyanide \\
\hline 4 & $\mathrm{C}_{2} \mathrm{H}_{4} \cdot \mathrm{H}^{+}$ & 29.0386 & \\
\hline 5 & $\mathrm{CH}_{3} \mathrm{~N} \cdot \mathrm{H}^{+}$ & 30.0338 & \\
\hline 6 & $\mathrm{CH}_{2} \mathrm{O} \cdot \mathrm{H}^{+}$ & 31.0178 & \\
\hline 7 & $\mathrm{O}_{2}^{+}$ & 31.9893 & \\
\hline 8 & $\mathrm{CH}_{5} \mathrm{~N} \cdot \mathrm{H}^{+}$ & 32.0495 & Methylamine \\
\hline 9 & $\mathrm{CH}_{4} \mathrm{O} \cdot \mathrm{H}^{+}$ & 33.0335 & \\
\hline 10 & $\mathrm{NH}_{3} \cdot \mathrm{H}_{2} \mathrm{O} \cdot \mathrm{H}^{+}$ & 36.0444 & \\
\hline 11 & $\left(\mathrm{H}_{2} \mathrm{O}\right)_{2} \cdot \mathrm{H}^{+}$ & 37.0284 & \\
\hline 12 & $\mathrm{C}_{2} \mathrm{H}_{3} \mathrm{~N} \cdot \mathrm{H}^{+}$ & 42.0338 & Acetonitrile \\
\hline 13 & $\mathrm{C}_{2} \mathrm{H}_{2} \mathrm{O} \cdot \mathrm{H}^{+}$ & 43.0178 & \\
\hline 14 & $\mathrm{C}_{2} \mathrm{H}_{5} \mathrm{~N} \cdot \mathrm{H}^{+}$ & 44.0495 & \\
\hline 15 & $\mathrm{C}_{2} \mathrm{H}_{4} \mathrm{O} \cdot \mathrm{H}^{+}$ & 45.0335 & \\
\hline 16 & $\mathrm{NO}_{2}^{+}$ & 45.9924 & \\
\hline 17 & $\mathrm{CH}_{3} \mathrm{NO} \cdot \mathrm{H}^{+}$ & 46.0287 & Formamide \\
\hline 18 & $\mathrm{C}_{2} \mathrm{H}_{7} \mathrm{~N} \cdot \mathrm{H}^{+}$ & 46.0651 & $\mathrm{C}_{2}$-Amine \\
\hline 19 & $\mathrm{C}_{2} \mathrm{~N}_{2} \cdot \mathrm{H}^{+}$ & 53.0134 & Cyanogen \\
\hline 20 & $\left(\mathrm{H}_{2} \mathrm{O}\right)_{3} \cdot \mathrm{H}^{+}$ & 55.0389 & \\
\hline 21 & $\mathrm{C}_{3} \mathrm{H}_{4} \mathrm{O} \cdot \mathrm{H}^{+}$ & 57.0335 & \\
\hline 22 & $\mathrm{C}_{4} \mathrm{H}_{8} \cdot \mathrm{H}^{+}$ & 57.0699 & \\
\hline 23 & $\mathrm{C}_{3} \mathrm{H}_{7} \mathrm{~N} \cdot \mathrm{H}^{+}$ & 58.0651 & \\
\hline 24 & $\mathrm{C}_{3} \mathrm{H}_{6} \mathrm{O} \cdot \mathrm{H}^{+}$ & 59.0491 & \\
\hline 25 & $\mathrm{C}_{2} \mathrm{H}_{5} \mathrm{NO} \cdot \mathrm{H}^{+}$ & 60.0444 & $\mathrm{C}_{2}$-Amide \\
\hline 26 & $\mathrm{C}_{3} \mathrm{H}_{9} \mathrm{~N} \cdot \mathrm{H}^{+}$ & 60.0808 & $\mathrm{C}_{3}$-Amine \\
\hline 27 & $\mathrm{CH}_{4} \mathrm{~N}_{2} \mathrm{O} \cdot \mathrm{H}^{+}$ & 61.0396 & \\
\hline 28 & $\mathrm{C}_{2} \mathrm{H}_{7} \mathrm{NO} \cdot \mathrm{H}^{+}$ & 62.0600 & 2-Aminoethanol \\
\hline 29 & $\mathrm{C}_{2} \mathrm{H}_{6} \mathrm{O}_{2} \cdot \mathrm{H}^{+}$ & 63.0441 & \\
\hline 30 & $\left(\mathrm{C}_{2} \mathrm{H}_{5} \mathrm{OH}\right) \cdot \mathrm{NH}_{3} \cdot \mathrm{H}^{+}$ & 64.0757 & \\
\hline 31 & $\left(\mathrm{C}_{2} \mathrm{H}_{5} \mathrm{OH}\right) \cdot \mathrm{H}_{2} \mathrm{O} \cdot \mathrm{H}^{+}$ & 65.0597 & \\
\hline 32 & $\mathrm{C}_{4} \mathrm{H}_{5} \mathrm{~N} \cdot \mathrm{H}^{+}$ & 68.0495 & Pyrrole \\
\hline 33 & $\mathrm{C}_{4} \mathrm{H}_{4} \mathrm{O} \cdot \mathrm{H}^{+}$ & 69.0335 & \\
\hline 34 & $\mathrm{C}_{5} \mathrm{H}_{8} \cdot \mathrm{H}^{+}$ & 69.0699 & \\
\hline 35 & $\mathrm{C}_{3} \mathrm{H}_{3} \mathrm{NO} \cdot \mathrm{H}^{+}$ & 70.0287 & \\
\hline 36 & $\mathrm{C}_{4} \mathrm{H}_{7} \mathrm{~N} \cdot \mathrm{H}^{+}$ & 70.0651 & 3-Pyrroline \\
\hline 37 & $\mathrm{C}_{3} \mathrm{H}_{2} \mathrm{O}_{2} \cdot \mathrm{H}^{+}$ & 71.0128 & \\
\hline 38 & $\mathrm{C}_{4} \mathrm{H}_{6} \mathrm{O} \cdot \mathrm{H}^{+}$ & 71.0491 & \\
\hline 39 & $\mathrm{C}_{3} \mathrm{H}_{5} \mathrm{NO} \cdot \mathrm{H}^{+}$ & 72.0444 & \\
\hline 40 & $\mathrm{C}_{4} \mathrm{H}_{9} \mathrm{~N} \cdot \mathrm{H}^{+}$ & 72.0808 & Pyrrolidine \\
\hline 41 & $\mathrm{C}_{3} \mathrm{H}_{4} \mathrm{O}_{2} \cdot \mathrm{H}^{+}$ & 73.0284 & \\
\hline 42 & $\mathrm{C}_{4} \mathrm{H}_{8} \mathrm{O} \cdot \mathrm{H}^{+}$ & 73.0648 & \\
\hline 43 & $\mathrm{C}_{3} \mathrm{H}_{7} \mathrm{NO} \cdot \mathrm{H}^{+}$ & 74.0600 & $\mathrm{C}_{3}$-Amide \\
\hline 44 & $\mathrm{C}_{4} \mathrm{H}_{11} \mathrm{~N} \cdot \mathrm{H}^{+}$ & 74.0964 & $\mathrm{C}_{4}$-Amine \\
\hline 45 & $\mathrm{C}_{4} \mathrm{H}_{10} \mathrm{O} \cdot \mathrm{H}^{+}$ & 75.0804 & \\
\hline 46 & $\mathrm{C}_{2} \mathrm{H}_{5} \mathrm{NO}_{2} \cdot \mathrm{H}^{+}$ & 76.0393 & Glycine \\
\hline 47 & $\mathrm{C}_{3} \mathrm{H}_{8} \mathrm{O}_{2} \cdot \mathrm{H}^{+}$ & 77.0597 & \\
\hline
\end{tabular}




\begin{tabular}{|c|c|c|c|}
\hline 48 & $\mathrm{C}_{2} \mathrm{H}_{7} \mathrm{NO}_{2} \cdot \mathrm{H}^{+}$ & 78.0549 & \\
\hline 49 & $\mathrm{CH}_{3} \mathrm{NH}_{2} \cdot\left(\mathrm{C}_{2} \mathrm{H}_{5} \mathrm{OH}\right) \cdot \mathrm{H}^{+}$ & 78.0913 & \\
\hline 50 & $\mathrm{C}_{2} \mathrm{H}_{6} \mathrm{OS} \cdot \mathrm{H}^{+}$ & 79.0212 & \\
\hline 51 & $\mathrm{C}_{6} \mathrm{H}_{6} \cdot \mathrm{H}^{+}$ & 79.0542 & \\
\hline 52 & $\mathrm{C}_{3} \mathrm{H}_{8} \mathrm{O} \cdot \mathrm{H}_{2} \mathrm{O} \cdot \mathrm{H}^{+}$ & 79.0753 & \\
\hline 53 & $\mathrm{C}_{5} \mathrm{H}_{5} \mathrm{~N} \cdot \mathrm{H}^{+}$ & 80.0495 & Pyridine \\
\hline 54 & $\mathrm{C}_{4} \mathrm{H}_{4} \mathrm{~N}_{2} \cdot \mathrm{H}^{+}$ & 81.0447 & Pyrimidine \\
\hline 55 & $\mathrm{C}_{2} \mathrm{H}_{6} \mathrm{O}_{2} \cdot \mathrm{H}_{2} \mathrm{O} \cdot \mathrm{H}^{+}$ & 81.0546 & \\
\hline 56 & $\mathrm{C}_{6} \mathrm{H}_{8} \cdot \mathrm{H}^{+}$ & 81.0699 & \\
\hline 57 & $\mathrm{C}_{5} \mathrm{H}_{7} \mathrm{~N} \cdot \mathrm{H}^{+}$ & 82.0651 & $N$-Methylpyrrole \\
\hline 58 & $\mathrm{C}_{2} \mathrm{H}_{6} \mathrm{O} \cdot\left(\mathrm{H}_{2} \mathrm{O}\right)_{2} \cdot \mathrm{H}^{+}$ & 83.0703 & \\
\hline 59 & $\mathrm{C}_{4} \mathrm{H}_{5} \mathrm{NO} \cdot \mathrm{H}^{+}$ & 84.0444 & \\
\hline 60 & $\mathrm{C}_{4} \mathrm{H}_{4} \mathrm{O}_{2} \cdot \mathrm{H}^{+}$ & 85.0284 & \\
\hline 61 & $\mathrm{C}_{5} \mathrm{H}_{8} \mathrm{O} \cdot \mathrm{H}^{+}$ & 85.0648 & \\
\hline 62 & $\mathrm{C}_{4} \mathrm{H}_{7} \mathrm{NO} \cdot \mathrm{H}^{+}$ & 86.0600 & \\
\hline 63 & $\mathrm{C}_{4} \mathrm{H}_{6} \mathrm{O}_{2} \cdot \mathrm{H}^{+}$ & 87.0441 & \\
\hline 64 & $\mathrm{C}_{5} \mathrm{H}_{10} \mathrm{O} \cdot \mathrm{H}^{+}$ & 87.0804 & \\
\hline 65 & $\mathrm{C}_{3} \mathrm{H}_{5} \mathrm{NO}_{2} \cdot \mathrm{H}^{+}$ & 88.0393 & $\mathrm{C}_{3}$-Oxoamide \\
\hline 66 & $\mathrm{C}_{4} \mathrm{H}_{9} \mathrm{NO} \cdot \mathrm{H}^{+}$ & 88.0757 & $\mathrm{C}_{4}$-Amide \\
\hline 67 & $\mathrm{C}_{5} \mathrm{H}_{13} \mathrm{~N} \cdot \mathrm{H}^{+}$ & 88.1121 & $\mathrm{C}_{5}$-Amine \\
\hline 68 & $\mathrm{C}_{3} \mathrm{H}_{4} \mathrm{O}_{3} \cdot \mathrm{H}^{+}$ & 89.0233 & \\
\hline 69 & $\mathrm{C}_{4} \mathrm{H}_{8} \mathrm{O}_{2} \cdot \mathrm{H}^{+}$ & 89.0597 & \\
\hline 70 & $\mathrm{C}_{5} \mathrm{H}_{12} \mathrm{O} \cdot \mathrm{H}^{+}$ & 89.0961 & \\
\hline 71 & $\mathrm{C}_{2} \mathrm{H}_{7} \mathrm{~N}_{3} \mathrm{O} \cdot \mathrm{H}^{+}$ & 90.0662 & \\
\hline 72 & $\mathrm{C}_{4} \mathrm{H}_{10} \mathrm{O}_{2} \cdot \mathrm{H}^{+}$ & 91.0754 & \\
\hline 73 & $\mathrm{CH}_{4} \mathrm{NO} \cdot\left(\mathrm{C}_{2} \mathrm{H}_{5} \mathrm{OH}\right) \cdot \mathrm{H}^{+}$ & 92.0706 & \\
\hline 74 & $\mathrm{C}_{2} \mathrm{H}_{7} \mathrm{~N} \cdot\left(\mathrm{C}_{2} \mathrm{H}_{5} \mathrm{OH}\right) \cdot \mathrm{H}^{+}$ & 92.1070 & \\
\hline 75 & $\mathrm{C}_{5} \mathrm{H}_{5} \mathrm{NO} \cdot \mathrm{H}^{+}$ & 96.0444 & \\
\hline 76 & $\mathrm{C}_{5} \mathrm{H}_{4} \mathrm{O}_{2} \cdot \mathrm{H}^{+}$ & 97.0284 & \\
\hline 77 & $\mathrm{C}_{6} \mathrm{H}_{8} \mathrm{O} \cdot \mathrm{H}^{+}$ & 97.0648 & \\
\hline 78 & $\mathrm{C}_{5} \mathrm{H}_{7} \mathrm{NO} \cdot \mathrm{H}^{+}$ & 98.0600 & \\
\hline 79 & $\mathrm{C}_{6} \mathrm{H}_{11} \mathrm{~N} \cdot \mathrm{H}^{+}$ & 98.0964 & 2,5-Dimethyl-3-pyrroline \\
\hline 80 & $\mathrm{C}_{5} \mathrm{H}_{6} \mathrm{O}_{2} \cdot \mathrm{H}^{+}$ & 99.0441 & \\
\hline 81 & $\mathrm{C}_{6} \mathrm{H}_{10} \mathrm{O} \cdot \mathrm{H}^{+}$ & 99.0804 & \\
\hline 82 & $\mathrm{C}_{5} \mathrm{H}_{9} \mathrm{NO} \cdot \mathrm{H}^{+}$ & 100.0757 & \\
\hline 83 & $\mathrm{C}_{5} \mathrm{H}_{8} \mathrm{O}_{2} \cdot \mathrm{H}^{+}$ & 101.0597 & \\
\hline 84 & $\mathrm{C}_{6} \mathrm{H}_{12} \mathrm{O} \cdot \mathrm{H}^{+}$ & 101.0961 & \\
\hline 85 & $\mathrm{C}_{4} \mathrm{H}_{7} \mathrm{NO}_{2} \cdot \mathrm{H}^{+}$ & 102.0550 & $\mathrm{C}_{4}$-Oxoamide \\
\hline 86 & $\mathrm{C}_{5} \mathrm{H}_{11} \mathrm{NO} \cdot \mathrm{H}^{+}$ & 102.0913 & $\mathrm{C}_{5}$-Amide \\
\hline 87 & $\mathrm{C}_{6} \mathrm{H}_{15} \mathrm{~N} \cdot \mathrm{H}^{+}$ & 102.1277 & $\mathrm{C}_{6}$-Amine \\
\hline 88 & $\mathrm{C}_{5} \mathrm{H}_{10} \mathrm{O}_{2} \cdot \mathrm{H}^{+}$ & 103.0754 & \\
\hline 89 & $\mathrm{C}_{6} \mathrm{H}_{14} \mathrm{O} \cdot \mathrm{H}^{+}$ & 103.1117 & \\
\hline 90 & $\mathrm{C}_{4} \mathrm{H}_{9} \mathrm{NO}_{2} \cdot \mathrm{H}^{+}$ & 104.0706 & 4-Aminobutyric acid \\
\hline 91 & $\mathrm{C}_{5} \mathrm{H}_{12} \mathrm{O}_{2} \cdot \mathrm{H}^{+}$ & 105.0910 & \\
\hline 92 & $\mathrm{C}_{2} \mathrm{H}_{6} \mathrm{NO} \cdot\left(\mathrm{C}_{2} \mathrm{H}_{5} \mathrm{OH}\right) \cdot \mathrm{H}^{+}$ & 106.0863 & \\
\hline 93 & $\mathrm{C}_{3} \mathrm{H}_{9} \mathrm{~N} \cdot\left(\mathrm{C}_{2} \mathrm{H}_{5} \mathrm{OH}\right) \cdot \mathrm{H}^{+}$ & 106.1226 & \\
\hline 94 & $\mathrm{C}_{6} \mathrm{H}_{5} \mathrm{NO} \cdot \mathrm{H}^{+}$ & 108.0444 & \\
\hline 95 & $\mathrm{C}_{8} \mathrm{H}_{12} \cdot \mathrm{H}^{+}$ & 109.1012 & \\
\hline
\end{tabular}




\begin{tabular}{|c|c|c|c|}
\hline 96 & $\mathrm{C}_{6} \mathrm{H}_{7} \mathrm{NO} \cdot \mathrm{H}^{+}$ & 110.0600 & \\
\hline 97 & $\mathrm{C}_{6} \mathrm{H}_{6} \mathrm{O}_{2} \cdot \mathrm{H}^{+}$ & 111.0441 & \\
\hline 98 & $\mathrm{C}_{6} \mathrm{H}_{8} \mathrm{O}_{2} \cdot \mathrm{H}^{+}$ & 113.0597 & \\
\hline 99 & $\mathrm{C}_{6} \mathrm{H}_{11} \mathrm{NO} \cdot \mathrm{H}^{+}$ & 114.0913 & \\
\hline 100 & $\mathrm{C}_{5} \mathrm{H}_{6} \mathrm{O}_{3} \cdot \mathrm{H}^{+}$ & 115.0390 & \\
\hline 101 & $\mathrm{C}_{6} \mathrm{H}_{10} \mathrm{O}_{2} \cdot \mathrm{H}^{+}$ & 115.0754 & \\
\hline 102 & $\mathrm{C}_{6} \mathrm{H}_{14} \mathrm{~N}_{2} \cdot \mathrm{H}^{+}$ & 115.1230 & 2,5-Dimethylpiperazine \\
\hline 103 & $\mathrm{C}_{5} \mathrm{H}_{9} \mathrm{NO}_{2} \cdot \mathrm{H}^{+}$ & 116.0760 & $\mathrm{C}_{5}$-Oxoamide \\
\hline 104 & $\mathrm{C}_{6} \mathrm{H}_{13} \mathrm{NO} \cdot \mathrm{H}^{+}$ & 116.1070 & $\mathrm{C}_{6}$-Amide \\
\hline 105 & $\mathrm{C}_{5} \mathrm{H}_{8} \mathrm{O}_{3} \cdot \mathrm{H}^{+}$ & 117.0546 & \\
\hline 106 & $\mathrm{C}_{6} \mathrm{H}_{12} \mathrm{O}_{2} \cdot \mathrm{H}^{+}$ & 117.0910 & \\
\hline 107 & $\mathrm{C}_{5} \mathrm{H}_{12} \mathrm{~N}_{2} \mathrm{O} \cdot \mathrm{H}^{+}$ & 117.1022 & \\
\hline 108 & $\mathrm{C}_{5} \mathrm{H}_{11} \mathrm{NO}_{2} \cdot \mathrm{H}^{+}$ & 118.0863 & L-Valine \\
\hline 109 & $\mathrm{C}_{4} \mathrm{H}_{10} \mathrm{~N}_{2} \mathrm{O}_{2} \cdot \mathrm{H}^{+}$ & 119.0815 & \\
\hline 110 & $\mathrm{C}_{6} \mathrm{H}_{14} \mathrm{O}_{2} \cdot \mathrm{H}^{+}$ & 119.1067 & \\
\hline 111 & $\mathrm{C}_{5} \mathrm{H}_{11} \mathrm{OS} \cdot \mathrm{H}^{+}$ & 120.0570 & \\
\hline 112 & $\mathrm{C}_{3} \mathrm{H}_{7} \mathrm{NO} \cdot\left(\mathrm{C}_{2} \mathrm{H}_{5} \mathrm{OH}\right) \cdot \mathrm{H}^{+}$ & 120.1019 & \\
\hline 113 & $\mathrm{C}_{4} \mathrm{H}_{12} \mathrm{~N} \cdot\left(\mathrm{C}_{2} \mathrm{H}_{5} \mathrm{OH}\right) \cdot \mathrm{H}^{+}$ & 120.1383 & \\
\hline 114 & $\mathrm{C}_{8} \mathrm{H}_{8} \mathrm{O} \cdot \mathrm{H}^{+}$ & 121.0648 & \\
\hline 115 & $\mathrm{C}_{6} \mathrm{H}_{14} \mathrm{O} \cdot \mathrm{H}_{2} \mathrm{O} \cdot \mathrm{H}^{+}$ & 121.1210 & \\
\hline 116 & $\mathrm{C}_{4} \mathrm{H}_{11} \mathrm{NOS} \cdot \mathrm{H}^{+}$ & 122.0634 & \\
\hline 117 & $\mathrm{C}_{8} \mathrm{H}_{11} \mathrm{~N} \cdot \mathrm{H}^{+}$ & 122.0964 & Phenethylamine \\
\hline 118 & $\mathrm{C}_{4} \mathrm{H}_{10} \mathrm{O}_{2} \mathrm{~S} \cdot \mathrm{H}^{+}$ & 123.0474 & \\
\hline 119 & $\mathrm{C}_{8} \mathrm{H}_{10} \mathrm{O} \cdot \mathrm{H}^{+}$ & 123.0804 & \\
\hline 120 & $\mathrm{C}_{9} \mathrm{H}_{14} \cdot \mathrm{H}^{+}$ & 123.1168 & \\
\hline 121 & $\mathrm{C}_{3} \mathrm{H}_{9} \mathrm{NO}_{2} \mathrm{~S} \cdot \mathrm{H}^{+}$ & 124.0995 & \\
\hline 122 & $\mathrm{C}_{7} \mathrm{H}_{8} \mathrm{O}_{2} \cdot \mathrm{H}^{+}$ & 125.0597 & \\
\hline 123 & $\mathrm{C}_{8} \mathrm{H}_{12} \mathrm{O} \cdot \mathrm{H}^{+}$ & 125.0961 & \\
\hline 124 & $\mathrm{C}_{6} \mathrm{H}_{6} \mathrm{O}_{3} \cdot \mathrm{H}^{+}$ & 127.0390 & \\
\hline 125 & $\mathrm{C}_{7} \mathrm{H}_{10} \mathrm{O}_{2} \cdot \mathrm{H}^{+}$ & 127.0753 & \\
\hline 126 & $\mathrm{C}_{8} \mathrm{H}_{14} \mathrm{O} \cdot \mathrm{H}^{+}$ & 127.1117 & \\
\hline 127 & $\mathrm{C}_{5} \mathrm{H}_{5} \mathrm{NO}_{3} \cdot \mathrm{H}^{+}$ & 128.0342 & \\
\hline 128 & $\mathrm{C}_{6} \mathrm{H}_{9} \mathrm{NO}_{2} \cdot \mathrm{H}^{+}$ & 128.0706 & \\
\hline 129 & $\mathrm{C}_{7} \mathrm{H}_{13} \mathrm{NO} \cdot \mathrm{H}^{+}$ & 128.1070 & \\
\hline 130 & $\mathrm{C}_{7} \mathrm{H}_{12} \mathrm{O}_{2} \cdot \mathrm{H}^{+}$ & 129.0910 & \\
\hline 131 & $\mathrm{C}_{9} \mathrm{H}_{7} \mathrm{~N} \cdot \mathrm{H}^{+}$ & 130.0651 & Quinoline \\
\hline 132 & $\mathrm{C}_{6} \mathrm{H}_{10} \mathrm{O}_{3} \cdot \mathrm{H}^{+}$ & 131.0702 & \\
\hline 133 & $\mathrm{C}_{7} \mathrm{H}_{14} \mathrm{O}_{2} \cdot \mathrm{H}^{+}$ & 131.1067 & \\
\hline 134 & $\mathrm{C}_{6} \mathrm{H}_{13} \mathrm{NO}_{2} \cdot \mathrm{H}^{+}$ & 132.1019 & L-Leucine \\
\hline 135 & $\mathrm{C}_{6} \mathrm{H}_{12} \mathrm{O}_{3} \cdot \mathrm{H}^{+}$ & 133.0859 & \\
\hline 136 & $\mathrm{C}_{3} \mathrm{H}_{5} \mathrm{NO}_{2} \cdot\left(\mathrm{C}_{2} \mathrm{H}_{5} \mathrm{OH}\right) \cdot \mathrm{H}^{+}$ & 134.0812 & \\
\hline 137 & $\mathrm{C}_{4} \mathrm{H}_{9} \mathrm{NO} \cdot\left(\mathrm{C}_{2} \mathrm{H}_{5} \mathrm{OH}\right) \cdot \mathrm{H}^{+}$ & 134.1176 & \\
\hline 138 & $\mathrm{C}_{5} \mathrm{H}_{13} \mathrm{~N} \cdot\left(\mathrm{C}_{2} \mathrm{H}_{5} \mathrm{OH}\right) \cdot \mathrm{H}^{+}$ & 134.1539 & \\
\hline 139 & $\mathrm{C}_{8} \mathrm{H}_{6} \mathrm{O}_{2} \cdot \mathrm{H}^{+}$ & 135.0441 & \\
\hline 140 & $\mathrm{C}_{4} \mathrm{H}_{10} \mathrm{~N}_{2} \mathrm{O}_{3} \cdot \mathrm{H}^{+}$ & 135.0764 & \\
\hline 141 & $\mathrm{C}_{6} \mathrm{H}_{14} \mathrm{O}_{3} \cdot \mathrm{H}^{+}$ & 135.1016 & \\
\hline 142 & $\mathrm{C}_{7} \mathrm{H}_{5} \mathrm{NS} \cdot \mathrm{H}^{+}$ & 136.0215 & \\
\hline 143 & $\mathrm{C}_{4} \mathrm{H}_{9} \mathrm{NO}_{4} \cdot \mathrm{H}^{+}$ & 136.0604 & \\
\hline
\end{tabular}




\begin{tabular}{|c|c|c|c|}
\hline 144 & $\mathrm{C}_{5} \mathrm{H}_{13} \mathrm{NO}_{3} \cdot \mathrm{H}^{+}$ & 136.0968 & \\
\hline 145 & $\mathrm{C}_{7} \mathrm{H}_{8} \mathrm{~N}_{2} \mathrm{O} \cdot \mathrm{H}^{+}$ & 137.0709 & \\
\hline 146 & $\mathrm{C}_{9} \mathrm{H}_{12} \mathrm{O} \cdot \mathrm{H}^{+}$ & 137.0961 & \\
\hline 147 & $\mathrm{C}_{6} \mathrm{H}_{14} \mathrm{O}_{2} \cdot \mathrm{H}_{2} \mathrm{O} \cdot \mathrm{H}^{+}$ & 137.1172 & \\
\hline 148 & $\mathrm{C}_{4} \mathrm{H}_{11} \mathrm{NO}_{2} \mathrm{~S} \cdot \mathrm{H}^{+}$ & 138.1039 & \\
\hline 149 & $\mathrm{C}_{7} \mathrm{H}_{8} \mathrm{O}_{3} \cdot \mathrm{H}^{+}$ & 141.0546 & \\
\hline 150 & $\mathrm{C}_{8} \mathrm{H}_{12} \mathrm{O}_{2} \cdot \mathrm{H}^{+}$ & 141.0910 & \\
\hline 151 & $\mathrm{C}_{9} \mathrm{H}_{16} \mathrm{O} \cdot \mathrm{H}^{+}$ & 141.1308 & \\
\hline 152 & $\mathrm{C}_{6} \mathrm{H}_{7} \mathrm{NO}_{3} \cdot \mathrm{H}^{+}$ & 142.0499 & \\
\hline 153 & $\mathrm{C}_{8} \mathrm{H}_{15} \mathrm{NO} \cdot \mathrm{H}^{+}$ & 142.1226 & \\
\hline 154 & $\mathrm{C}_{7} \mathrm{H}_{10} \mathrm{O}_{3} \cdot \mathrm{H}^{+}$ & 143.0703 & \\
\hline 155 & $\mathrm{C}_{8} \mathrm{H}_{14} \mathrm{O}_{2} \cdot \mathrm{H}^{+}$ & 143.1067 & \\
\hline 156 & $\mathrm{C}_{8} \mathrm{H}_{18} \mathrm{~N}_{2} \cdot \mathrm{H}^{+}$ & 143.1542 & 1-Butylpiperazine \\
\hline 157 & $\mathrm{C}_{5} \mathrm{H}_{9} \mathrm{~N}_{3} \mathrm{O}_{2} \cdot \mathrm{H}^{+}$ & 144.0768 & \\
\hline 158 & $\mathrm{C}_{7} \mathrm{H}_{13} \mathrm{NO}_{2} \cdot \mathrm{H}^{+}$ & 144.1019 & \\
\hline 159 & $\mathrm{C}_{8} \mathrm{H}_{17} \mathrm{NO} \cdot \mathrm{H}^{+}$ & 144.1383 & \\
\hline 160 & $\mathrm{C}_{6} \mathrm{H}_{8} \mathrm{O}_{4} \cdot \mathrm{H}^{+}$ & 145.0495 & \\
\hline 161 & $\mathrm{C}_{7} \mathrm{H}_{12} \mathrm{O}_{3} \cdot \mathrm{H}^{+}$ & 145.0859 & \\
\hline 162 & $\mathrm{C}_{8} \mathrm{H}_{16} \mathrm{O}_{2} \cdot \mathrm{H}^{+}$ & 145.1223 & \\
\hline 163 & $\mathrm{C}_{6} \mathrm{H}_{11} \mathrm{NO}_{3} \cdot \mathrm{H}^{+}$ & 146.0811 & \\
\hline 164 & $\mathrm{C}_{7} \mathrm{H}_{15} \mathrm{NO}_{2} \cdot \mathrm{H}^{+}$ & 146.1176 & 7-Aminoheptanoic acid \\
\hline 165 & $\mathrm{C}_{6} \mathrm{H}_{10} \mathrm{O}_{4} \cdot \mathrm{H}^{+}$ & 147.0652 & \\
\hline 166 & $\mathrm{C}_{7} \mathrm{H}_{14} \mathrm{O}_{3} \cdot \mathrm{H}^{+}$ & 147.1016 & \\
\hline 167 & $\mathrm{C}_{8} \mathrm{H}_{18} \mathrm{O}_{2} \cdot \mathrm{H}^{+}$ & 147.1379 & \\
\hline 168 & $\mathrm{C}_{4} \mathrm{H}_{7} \mathrm{NO}_{2} \cdot\left(\mathrm{C}_{2} \mathrm{H}_{5} \mathrm{OH}\right) \cdot \mathrm{H}^{+}$ & 148.0968 & \\
\hline 169 & $\mathrm{C}_{5} \mathrm{H}_{11} \mathrm{NO} \cdot\left(\mathrm{C}_{2} \mathrm{H}_{5} \mathrm{OH}\right) \cdot \mathrm{H}^{+}$ & 148.1332 & \\
\hline 170 & $\mathrm{C}_{6} \mathrm{H}_{15} \mathrm{~N} \cdot\left(\mathrm{C}_{2} \mathrm{H}_{5} \mathrm{OH}\right) \cdot \mathrm{H}^{+}$ & 148.1696 & \\
\hline 171 & $\mathrm{C}_{5} \mathrm{H}_{8} \mathrm{O}_{3} \mathrm{~S} \cdot \mathrm{H}^{+}$ & 149.0267 & \\
\hline 172 & $\mathrm{C}_{9} \mathrm{H}_{8} \mathrm{O}_{2} \cdot \mathrm{H}^{+}$ & 149.0597 & \\
\hline 173 & $\mathrm{C}_{6} \mathrm{H}_{12} \mathrm{O}_{4} \cdot \mathrm{H}^{+}$ & 149.0808 & \\
\hline 174 & $\mathrm{C}_{8} \mathrm{H}_{7} \mathrm{NS} \cdot \mathrm{H}^{+}$ & 150.0372 & \\
\hline 175 & $\mathrm{C}_{5} \mathrm{H}_{11} \mathrm{NO}_{4} \cdot \mathrm{H}^{+}$ & 150.0761 & \\
\hline 176 & $\mathrm{C}_{5} \mathrm{H}_{15} \mathrm{~N}_{3} \mathrm{O}_{2} \cdot \mathrm{H}^{+}$ & 150.1237 & \\
\hline 177 & $\mathrm{C}_{5} \mathrm{H}_{14} \mathrm{~N}_{2} \mathrm{OS} \cdot \mathrm{H}^{+}$ & 151.0899 & \\
\hline 178 & $\mathrm{C}_{10} \mathrm{H}_{14} \mathrm{O} \cdot \mathrm{H}^{+}$ & 151.1117 & \\
\hline 179 & $\mathrm{C}_{7} \mathrm{H}_{5} \mathrm{NOS} \cdot \mathrm{H}^{+}$ & 152.0165 & \\
\hline 180 & $\mathrm{C}_{10} \mathrm{H}_{15} \mathrm{O} \cdot \mathrm{H}^{+}$ & 152.1196 & \\
\hline 181 & $\mathrm{C}_{6} \mathrm{H}_{14} \mathrm{O}_{3} \cdot \mathrm{H}_{2} \mathrm{O} \cdot \mathrm{H}^{+}$ & 153.1121 & \\
\hline 182 & $\mathrm{C}_{9} \mathrm{H}_{14} \mathrm{O}_{2} \cdot \mathrm{H}^{+}$ & 155.1067 & \\
\hline 183 & $\mathrm{C}_{9} \mathrm{H}_{18} \mathrm{~N}_{2} \cdot \mathrm{H}^{+}$ & 155.1543 & 4-Pyrrolidinopiperidine \\
\hline 184 & $\mathrm{C}_{8} \mathrm{H}_{12} \mathrm{O}_{3} \cdot \mathrm{H}^{+}$ & 157.0859 & \\
\hline 185 & $\mathrm{C}_{9} \mathrm{H}_{16} \mathrm{O}_{2} \cdot \mathrm{H}^{+}$ & 157.1223 & \\
\hline 186 & $\mathrm{C}_{9} \mathrm{H}_{20} \mathrm{~N}_{2} \cdot \mathrm{H}^{+}$ & 157.1699 & Triacetonediamine \\
\hline 187 & $\mathrm{C}_{11} \mathrm{H}_{11} \mathrm{~N} \cdot \mathrm{H}^{+}$ & 158.0964 & 2,8-Dimethylquinoline \\
\hline 188 & $\mathrm{C}_{9} \mathrm{H}_{19} \mathrm{NO} \cdot \mathrm{H}^{+}$ & 158.1539 & \\
\hline 189 & $\mathrm{C}_{7} \mathrm{H}_{10} \mathrm{O}_{4} \cdot \mathrm{H}^{+}$ & 159.0652 & \\
\hline 190 & $\mathrm{C}_{8} \mathrm{H}_{14} \mathrm{O}_{3} \cdot \mathrm{H}^{+}$ & 159.1016 & \\
\hline 191 & $\mathrm{C}_{10} \mathrm{H}_{9} \mathrm{NO} \cdot \mathrm{H}^{+}$ & 160.0757 & \\
\hline
\end{tabular}




\begin{tabular}{|l|c|c|c|}
\hline 192 & $\mathrm{C}_{11} \mathrm{H}_{13} \mathrm{~N} \cdot \mathrm{H}^{+}$ & 160.1121 & 2,3,3-Trimethylindolenine \\
\hline 193 & $\mathrm{C}_{8} \mathrm{H}_{17} \mathrm{NO}_{2} \cdot \mathrm{H}^{+}$ & 160.1332 & 8-Aminooctanoic acid \\
\hline 194 & $\mathrm{C}_{7} \mathrm{H}_{12} \mathrm{O}_{4} \cdot \mathrm{H}^{+}$ & 161.0808 & \\
\hline 195 & $\mathrm{C}_{8} \mathrm{H}_{16} \mathrm{O}_{3} \cdot \mathrm{H}^{+}$ & 161.1172 & \\
\hline 196 & $\mathrm{C}_{9} \mathrm{H}_{20} \mathrm{O}_{2} \cdot \mathrm{H}^{+}$ & 161.1536 & \\
\hline 197 & $\mathrm{C}_{4} \mathrm{H}_{5} \mathrm{NO}_{3} \cdot\left(\mathrm{C}_{2} \mathrm{H}_{5} \mathrm{OH}\right) \cdot \mathrm{H}^{+}$ & 162.0761 & \\
\hline 198 & $\mathrm{C}_{5} \mathrm{H}_{9} \mathrm{NO}_{2} \cdot\left(\mathrm{C}_{2} \mathrm{H}_{5} \mathrm{OH}\right) \cdot \mathrm{H}^{+}$ & 162.1125 & \\
\hline 199 & $\mathrm{C}_{6} \mathrm{H}_{13} \mathrm{NO} \cdot\left(\mathrm{C}_{2} \mathrm{H}_{5} \mathrm{OH}\right) \cdot \mathrm{H}^{+}$ & 162.1489 & \\
\hline 200 & $\mathrm{C}_{8} \mathrm{H}_{18} \mathrm{O}_{3} \cdot \mathrm{H}^{+}$ & 163.1329 & \\
\hline
\end{tabular}




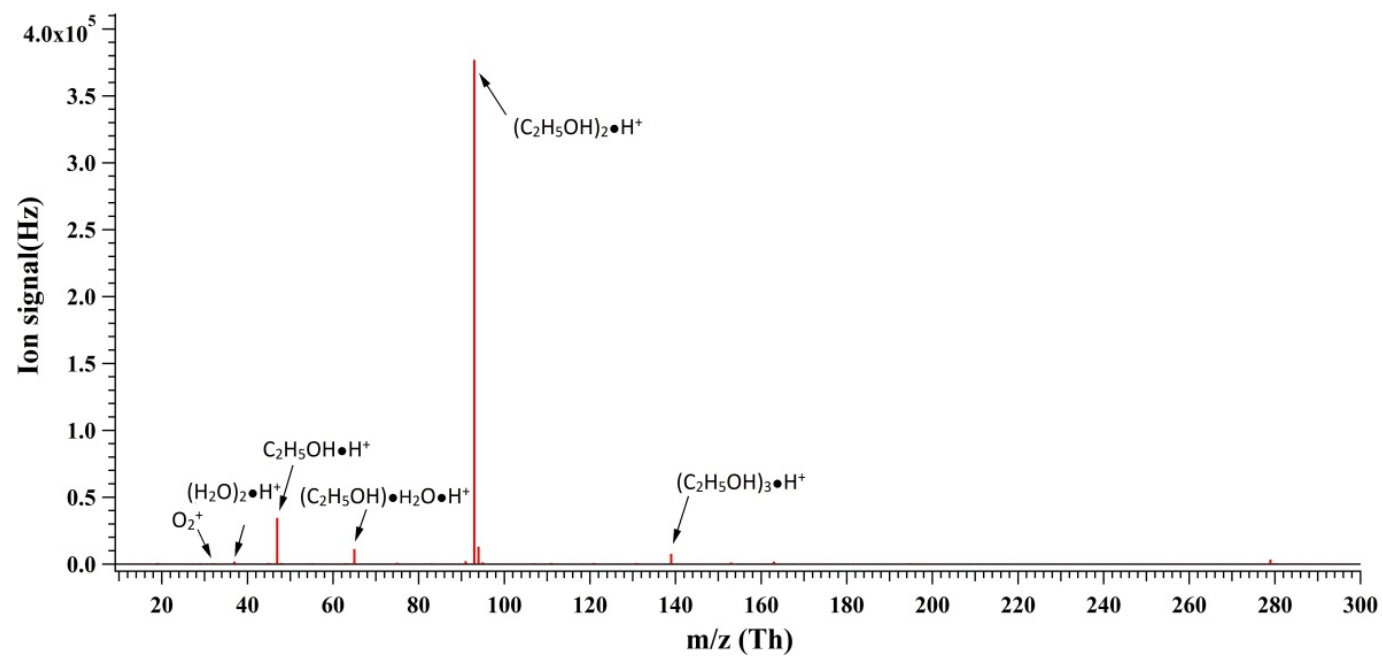

Figure S1. A typical mass spectrum with $\mathrm{RH}<20 \%$. The dominant reagent ions are protonated ethanol dimer $\left(\left(\mathrm{C}_{2} \mathrm{H}_{5} \mathrm{OH}\right)_{2} \cdot \mathrm{H}^{+}\right)$, monomer $\left(\left(\mathrm{C}_{2} \mathrm{H}_{5} \mathrm{OH}\right) \cdot \mathrm{H}^{+}\right)$, and trimer $\left(\left(\mathrm{C}_{2} \mathrm{H}_{5} \mathrm{OH}\right)_{3} \cdot \mathrm{H}^{+}\right)$. The ratio of the clusters of protonated ethanol with water $\left(\mathrm{C}_{2} \mathrm{H}_{5} \mathrm{OH} \cdot \mathrm{H}_{2} \mathrm{O} \cdot \mathrm{H}^{+}\right)$ to the sum of $\left(\mathrm{C}_{2} \mathrm{H}_{5} \mathrm{OH}\right) \cdot \mathrm{H}^{+},\left(\mathrm{C}_{2} \mathrm{H}_{5} \mathrm{OH}\right)_{2} \cdot \mathrm{H}^{+}$, and $\left(\mathrm{C}_{2} \mathrm{H}_{5} \mathrm{OH}\right)_{3} \cdot \mathrm{H}^{+}$is $\sim 0.026$. 

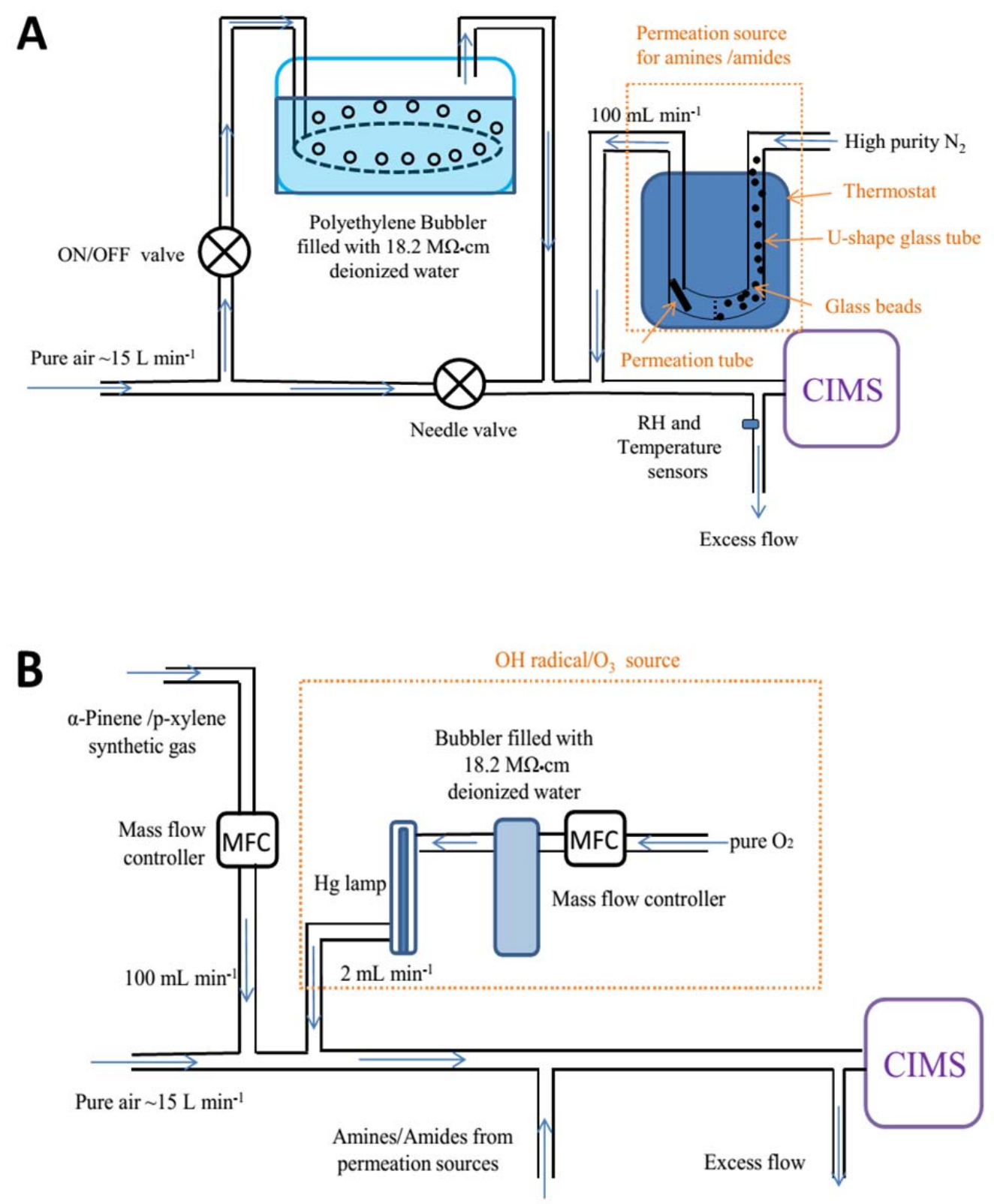

Figure S2. Schematics for laboratory tests of effects of (A) RH and (B) organics. All sampling lines are made of PFA or PTFE material. 


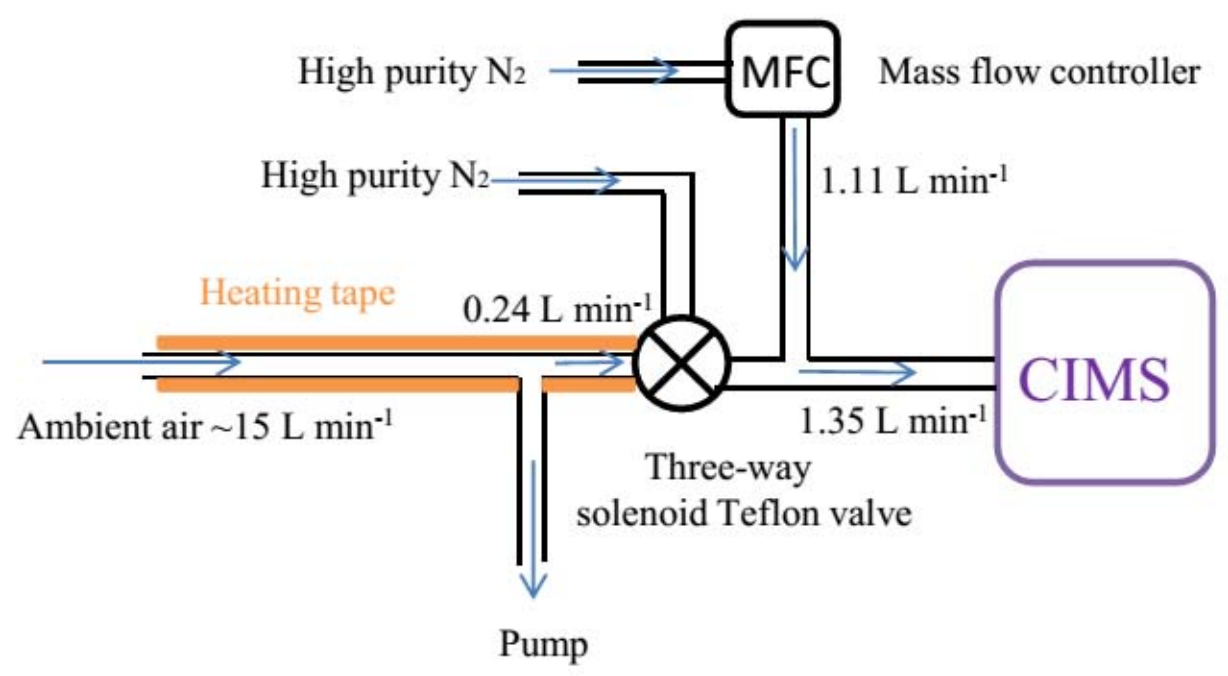

Figure S3. Schematic of CIMS setup during the field measurements. 


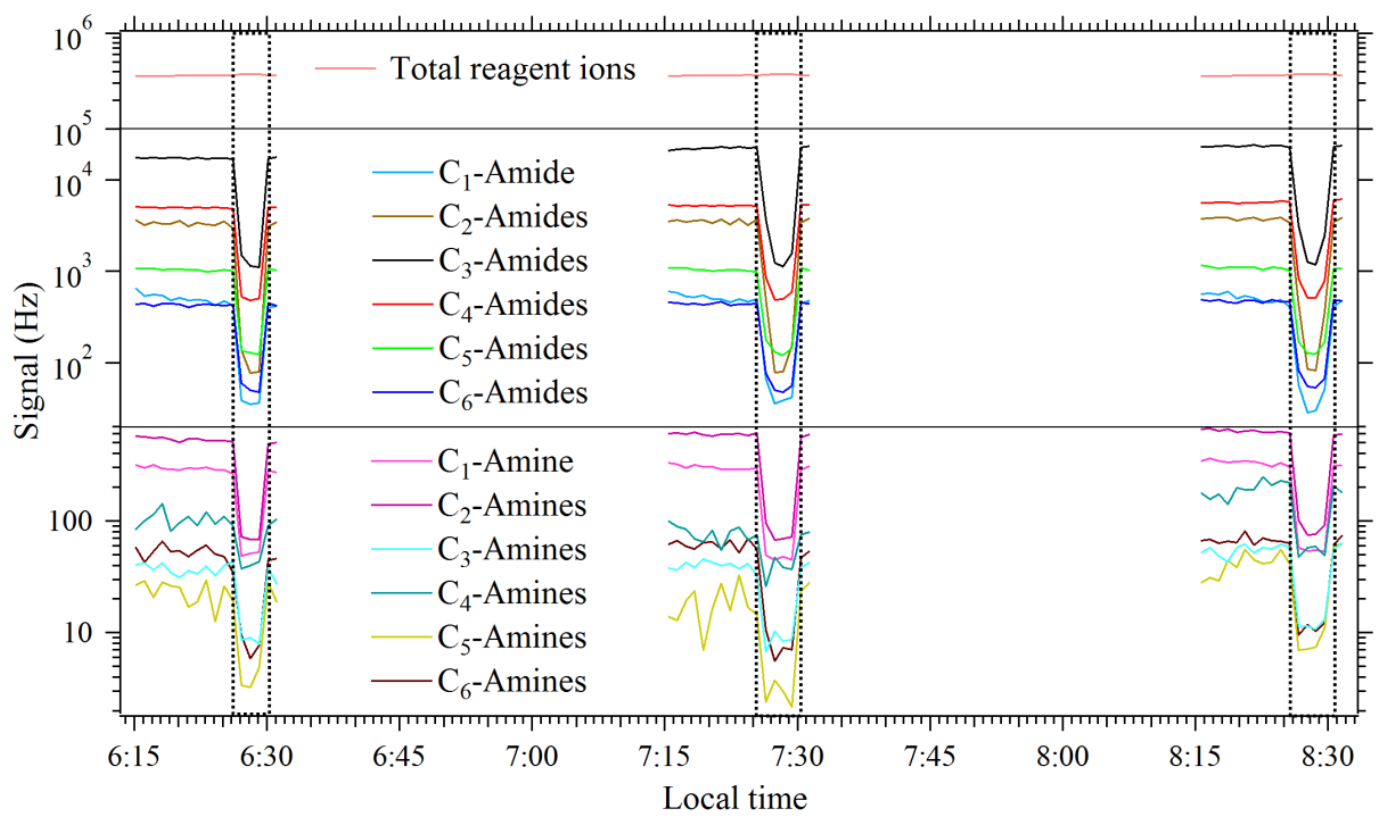

Figure S4. Background check for amines and amides during a $3 \mathrm{~h}$ ambient sampling period. 


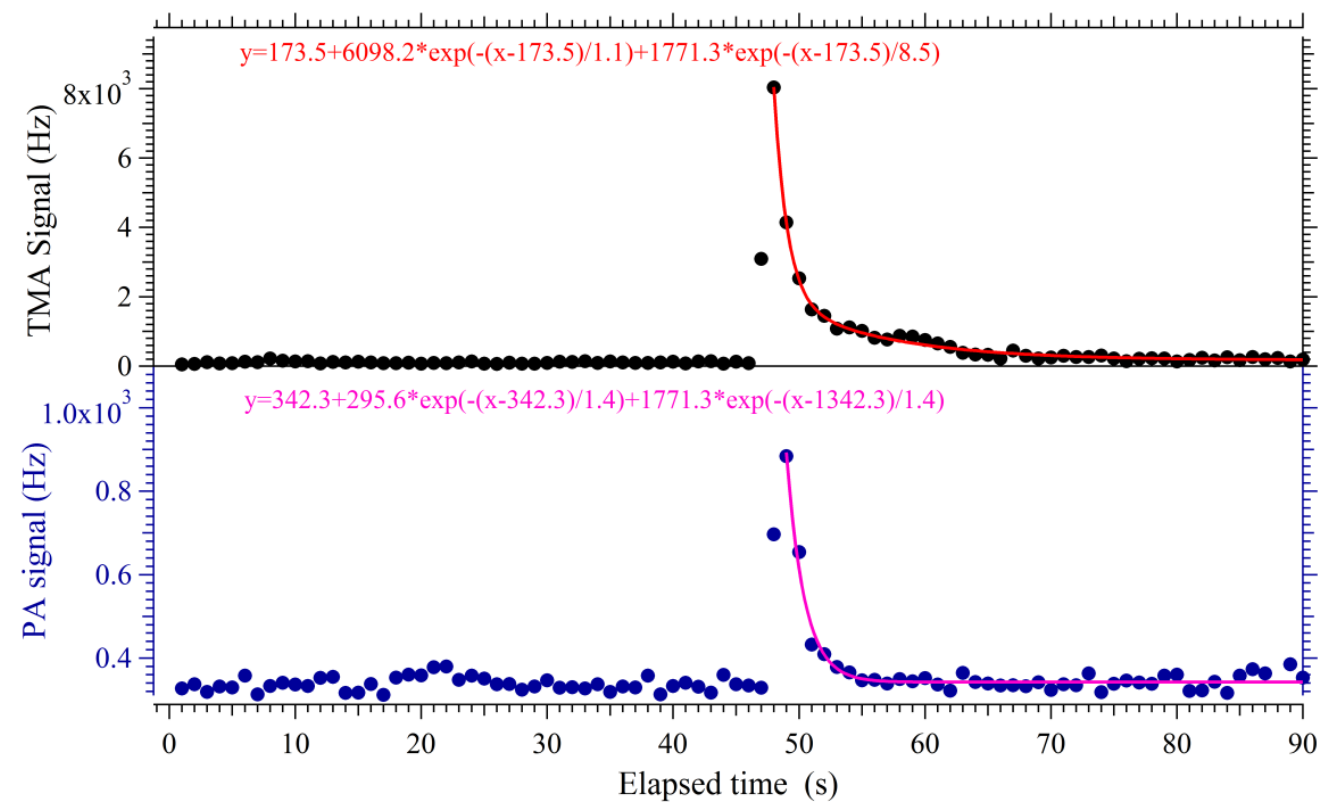

Figure S5. Inlet memory of TMA and PA by inlet spike tests. The red and pink curves are fittings by the sum of two decaying exponentials. The characteristic decaying times of two exponentials, which are displacement of amines and amides inside the inlet by pumping and removing amines and amides adsorbed on the inlet surface, were $1.1 \mathrm{~s}$ and $8.5 \mathrm{~s}$ for TMA, and $1.4 \mathrm{~s}$ and $1.4 \mathrm{~s}$ for PA, respectively. 


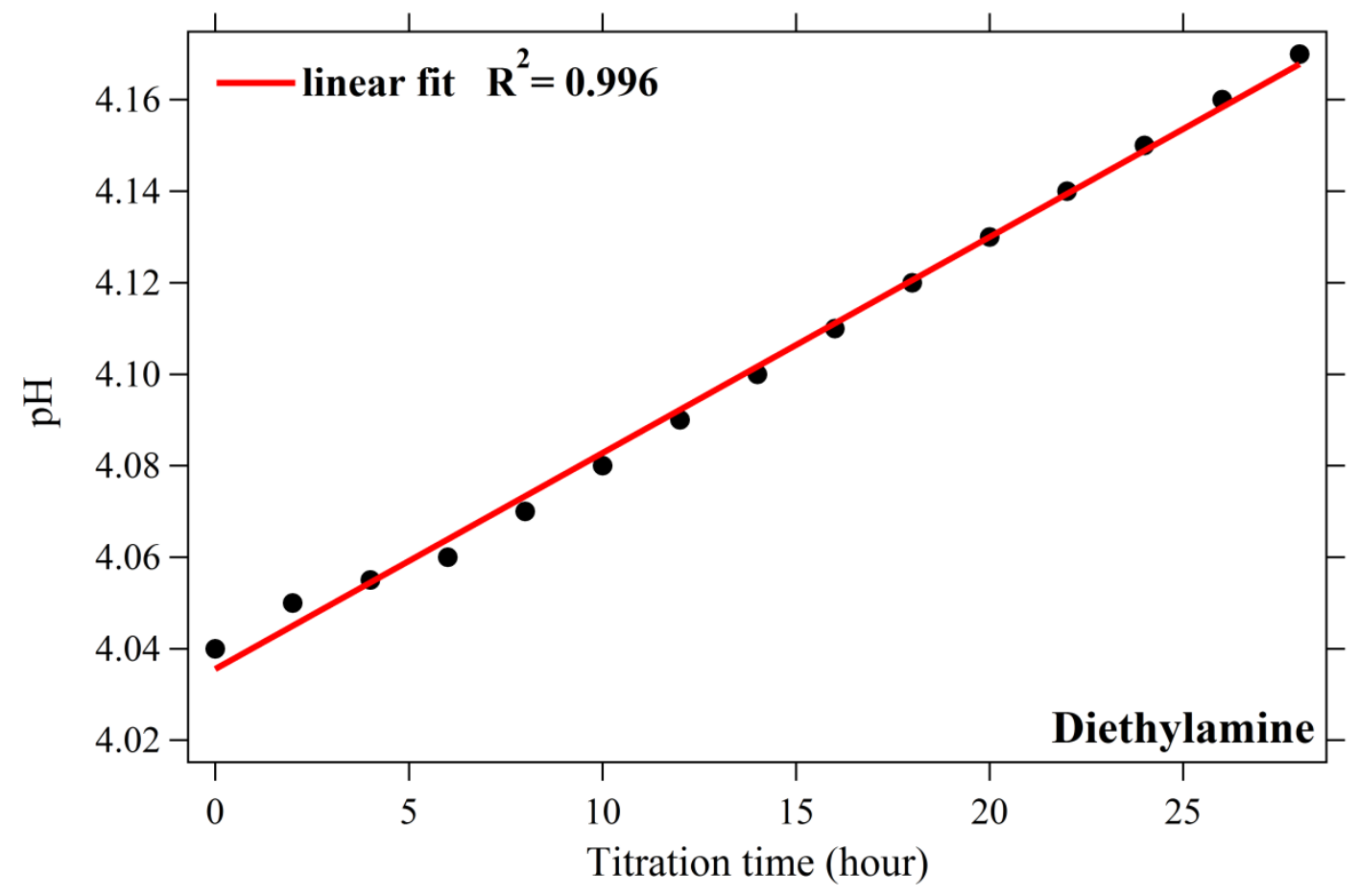

Figure S6. Changes in $\mathrm{pH}$ values of $\mathrm{HNO}_{3}$ solution as titration by diethylamine proceeds. Note that $\mathrm{pH}$ values in this plot are 2-h averages 

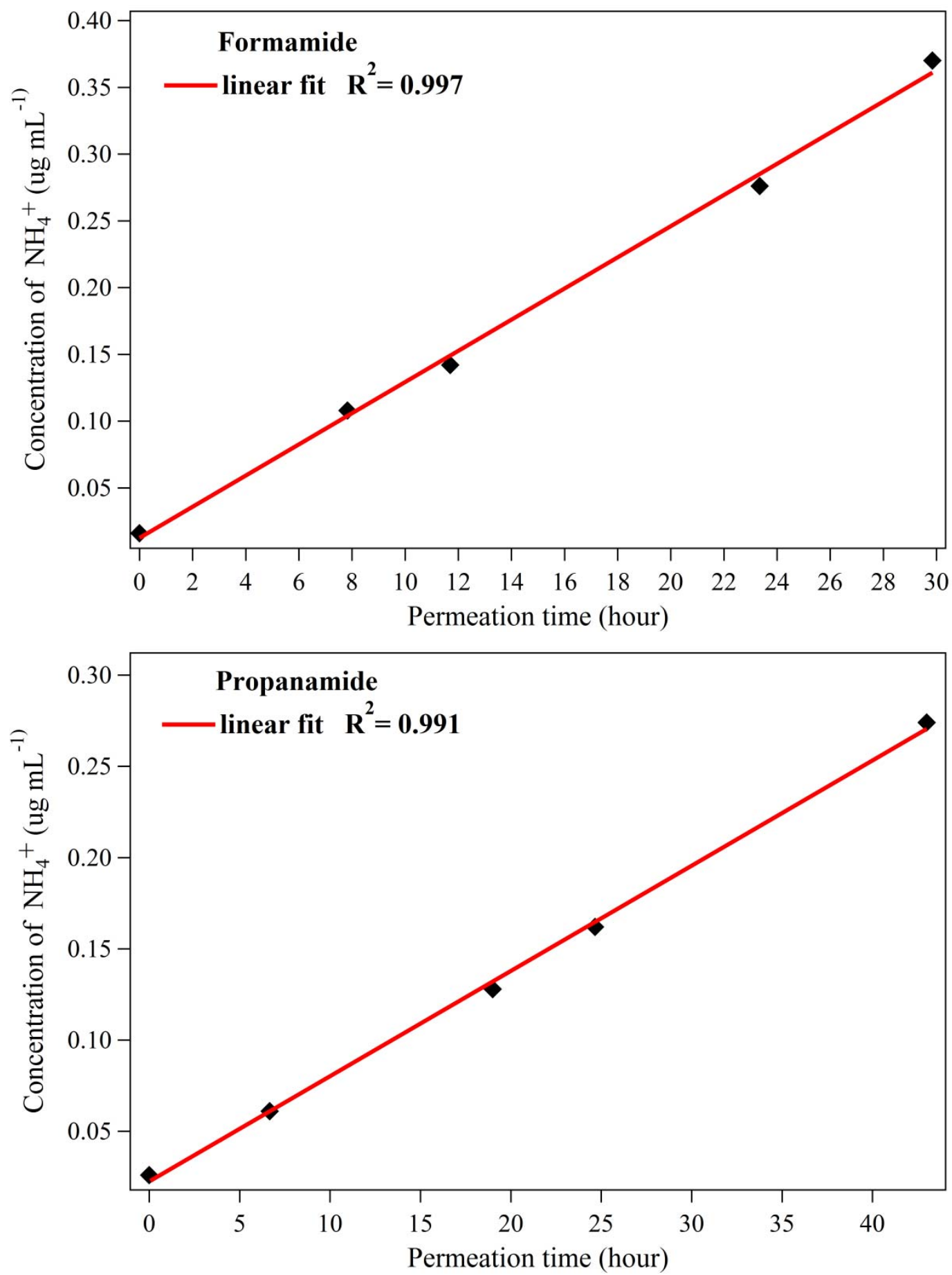

Figure S7. Changes in $\mathrm{NH}_{4}{ }^{+}$concentration as hydrolysis of formamide and propanamide proceeds. 

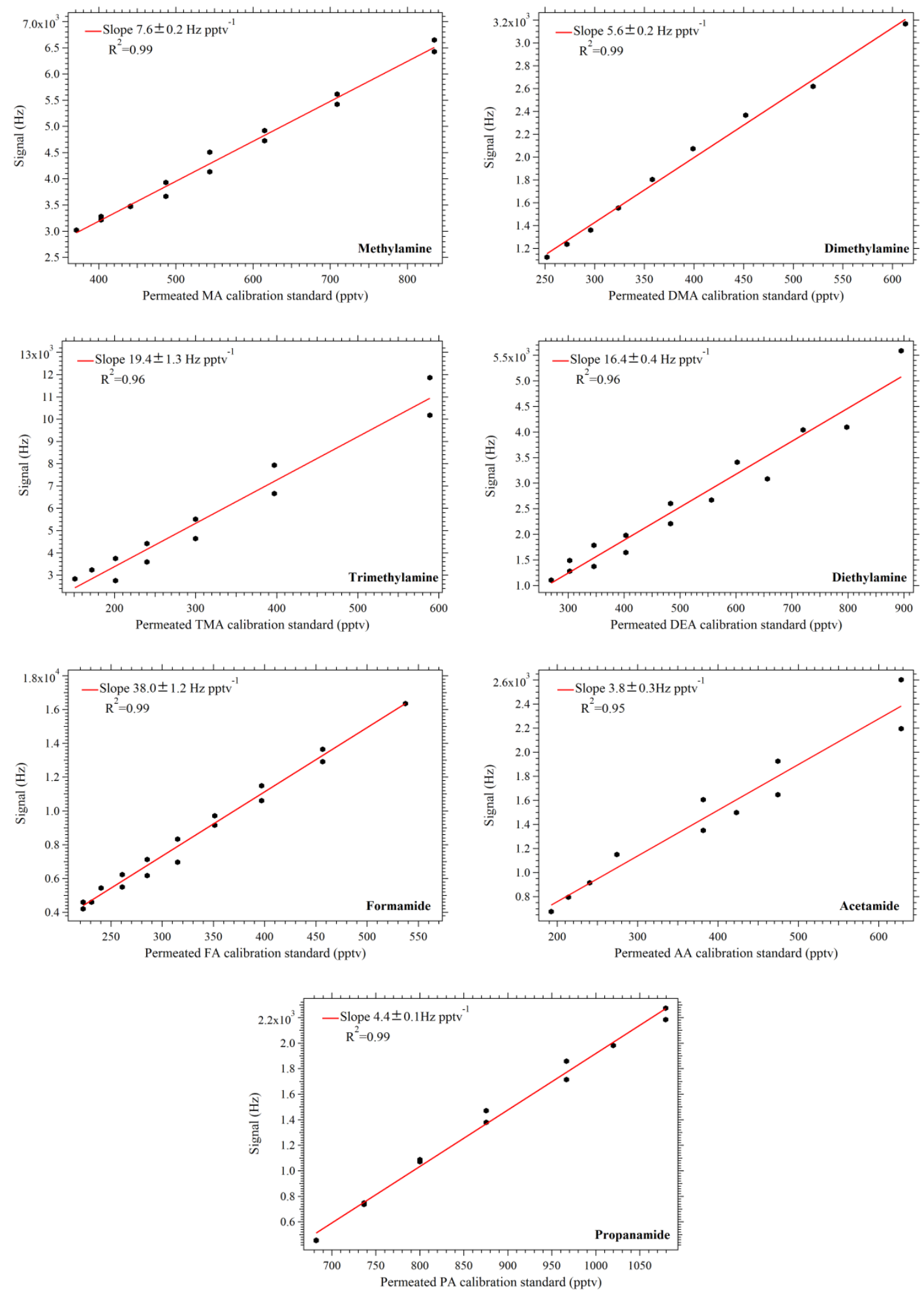

Figure S8. Calibration curves of $\mathrm{C}_{1}$ - to $\mathrm{C}_{4}$-amines (MA, DMA, TMA, and DEA) and $\mathrm{C}_{1}$ - to $\mathrm{C}_{3}$-amides (FA, AA, and PA). The red solid lines are linear fittings to guide the eye and the slopes are sensitivities for detection. 

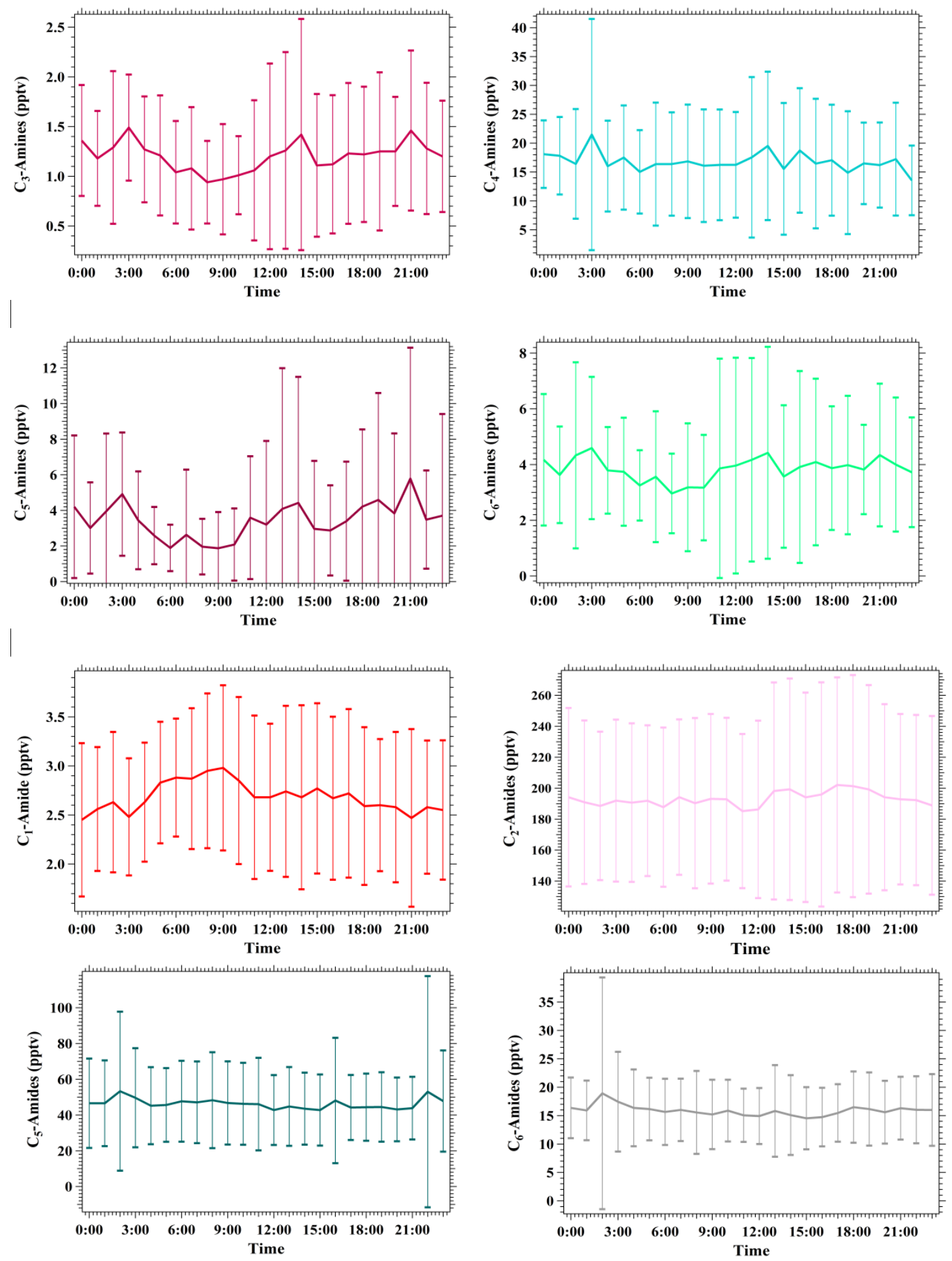

Figure S9. Diurnal variations of amines and amides with less variation. 

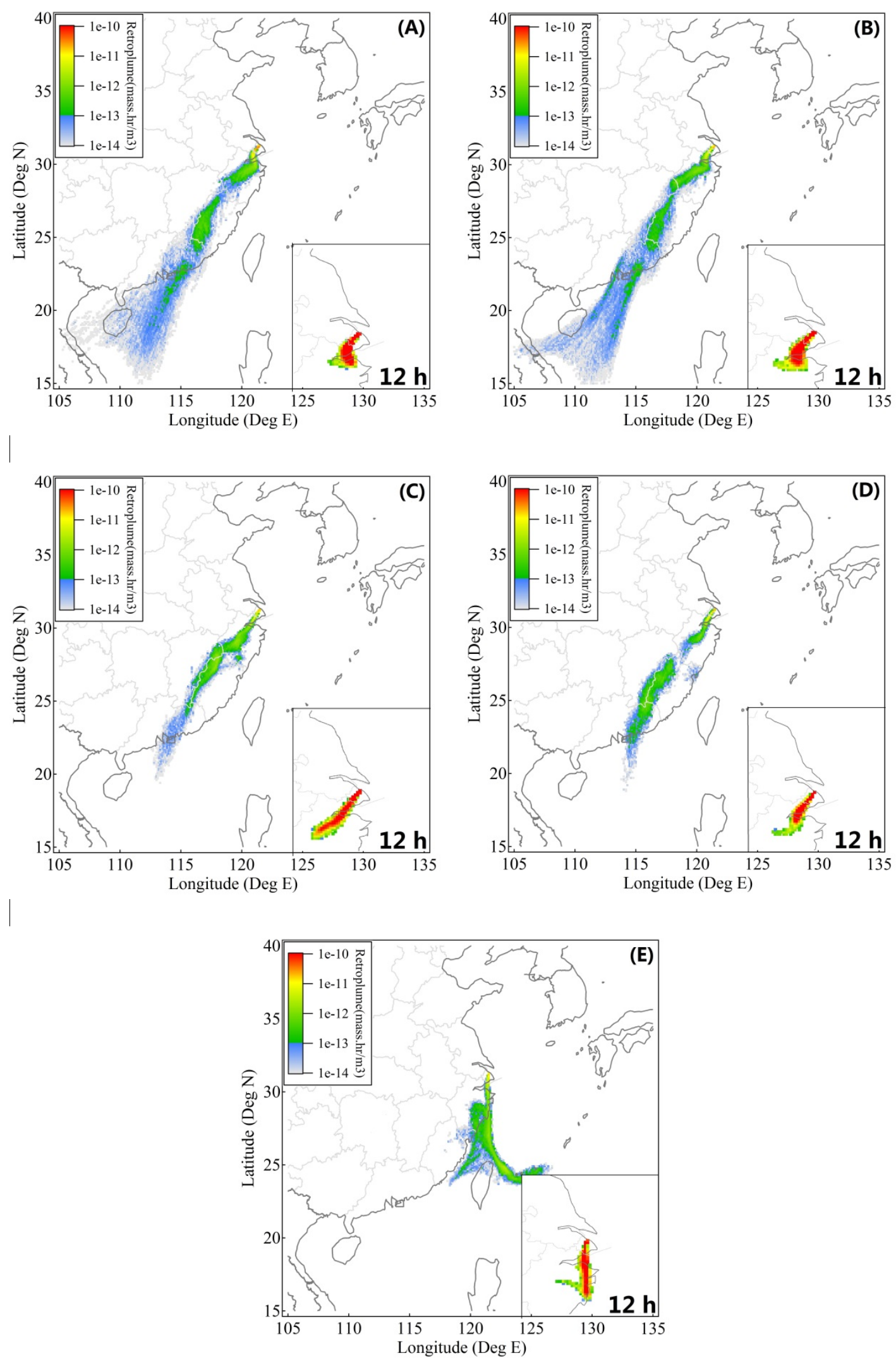

Figure S10. Three-day backward retroplumes (100 m above the ground level) from the sampling location at (A) 07:00, 27 July 2015; (B) 07:00, 28 July 2015; (C) 07:00, 30 July 2015; (D) 07:00, 31 July 2015; and (E) 07:00, 4 August 2015. The embedded boxes show 12h backward trajectories. 